University of Texas at El Paso

ScholarWorks@UTEP

$12-2010$

\title{
Reducing Over-Conservative Expert Failure Rate Estimates in the Presence of Limited Data: A New Probabilistic/Fuzzy Approach
}

\author{
Carlos M. Ferregut \\ The University of Texas at El Paso, ferregut@utep.edu \\ F. Joshua Campos \\ The University of Texas at El Paso, fjcampos@miners.utep.edu \\ Vladik Kreinovich \\ The University of Texas at El Paso, vladik@utep.edu
}

Follow this and additional works at: https://scholarworks.utep.edu/cs_techrep

Part of the Computer Engineering Commons

Comments:

Technical Report: UTEP-CS-10-33a

Published in Proceedings of the 30th Annual Conference of the North American Fuzzy

Information Processing Society NAFIPS'2011, El Paso, Texas, March 18-20, 2011.

\section{Recommended Citation}

Ferregut, Carlos M.; Campos, F. Joshua; and Kreinovich, Vladik, "Reducing Over-Conservative Expert Failure Rate Estimates in the Presence of Limited Data: A New Probabilistic/Fuzzy Approach" (2010). Departmental Technical Reports (CS). 674.

https://scholarworks.utep.edu/cs_techrep/674

This Article is brought to you for free and open access by the Computer Science at ScholarWorks@UTEP. It has been accepted for inclusion in Departmental Technical Reports (CS) by an authorized administrator of ScholarWorks@UTEP.For more information, please contact Iweber@utep.edu. 


\title{
Towards Optimal Sensor Placement in Multi-Zone Measurements
}

\author{
Octavio Lerma, Craig Tweedie, and Vladik Kreinovich \\ Cyber-ShARE Center \\ University of Texas at El Paso \\ $500 \mathrm{~W}$. University \\ El Paso, TX 79968, USA \\ Emails lolerma@episd.org, ctweedie@utep.edu, vladik@utep.edu
}

\begin{abstract}
In multi-zone areas, where the boundaries change with time, it is desirable to place sensors in such a way that the boundary is covered at all times. In this paper, we describe the optimal sensor placement with this property. In this optimal placement, sensors are placed along a see-saw trajectory going between the current location of the boundary and its farthest future location.
\end{abstract}

\section{Practical Problem: To Determine the Motion of THE INTER-ZONE BOUNDARY}

Practical need for measurements at different spatial locations. In many areas such as weather prediction, the partial differential equations that can be used for the prediction are known. To make accurate predictions, we need to have a very accurate picture of the initial conditions, a picture that describes, in detail, current temperature, atmospheric pressure, wind, and other characteristics at different spatial locations. To find the values of these characteristics, we need to place sensors at these locations and use these sensors to perform the corresponding measurements.

Need for sensor placement. In some areas - e.g., in and around big cities - there is usually a large number of sensors, many of them operated by volunteers who place these sensors in their homes and who regularly upload the readings of these sensors to the corresponding open-access web-based data networks. However, in other areas (e.g., in the Arctic), the existing sensor coverage is too sparse, so more sensors are needed.

The placement of new sensors not only helps in achieving short-term goals such as weather predictions, it also helps in analyzing long-term effects such as climate and environmental changes.

Need for optimal sensor placements. Placement and maintenance of sensors in remote areas is often costly, so it is desirable to come up with the optimal ways to place sensors - so that we can achieve the desired accuracy and coverage at the smallest possible cost.

Case of homogeneous (single-zone) regions. The problem of optimal sensor placement is simpler for homogeneous (singlezone) regions, in which the measured quantity smoothly changes from one location to another. A value $v_{i}$ measured by the $i$-th sensor represents - reasonably accurately - the value $v\left(x_{i}\right)$ of the desired quantity $v$ at this sensor's location $x_{i}$. This measured value $v_{i}$ is also a good approximation to the value $v(x)$ of this quantity at locations $x$ which are close to the sensor's location $x_{i}: v(x) \approx v_{i}=v\left(x_{i}\right)$. The closer the location $x$ to the sensor's location $x_{i}$, the better this approximation. Vice versa, the farther away the location $x$ is from the sensor $x_{i}$, the less accurate is the resulting approximation $v(x) \approx v_{i}=v\left(x_{i}\right)$.

Suppose that we have placed sensors at locations $x_{1}, \ldots, x_{n}$. Then, after we get the measurement results $v_{1}=$ $v\left(x_{1}\right), \ldots, v_{n}=v\left(x_{n}\right)$ from all these sensors, we can, for every spatial location $x$, approximate the value $v(x)$ by the result $v\left(x_{i}\right)$ measured by the closest sensor $i$.

In general, for every spatial location $x$, the closer this location is to one of the sensor's locations $x_{1}, \ldots, x_{n}$, the more accurate this approximation - and thus, the more accurately we can determine the value $v(x)$.

Our objective is to determine the values $v(x)$ for all spatial locations with a certain accuracy. Thus, the quality of a sensor network can be measured by accuracy with which it determines $v(x)$ at all spatial locations $x$. From this viewpoint, the quality of a sensor network is determined by the "worst" spatial location - i.e., by a spatial location which is the farthest away from all the sensors (and for which, therefore, the approximation accuracy is the worst).

With respect to this criterion, it does not make sense to place sensors more densely in one subregion - because spreading them to locations with the worst coverage will improve the overall quality. In other words, for such homogeneous regions, the optimal sensor placement should be uniformly distributed throughout the region; see, e.g., [1], [2], [3].

Case of multi-zone measurements: we need to determine the motion of the inter-zone boundaries. In practice, regions are often not homogeneous, they consist of several distinct zones with a sharp boundary between the zones. The simplest example is a shoreline - the boundary between the land and the ocean. In mountain regions, there is also a sharp lines between a glacier and the grassy zone around it, etc.

For such multi-zone areas, we need not only to find the values of the desired characteristics at different zones, we also need to get a good understanding of the exact location of the 
boundary between the zones.

Of course, if the boundary was absolutely stable, then we would be able to map it once - and there would be no need to continue monitoring the boundary by using sensors. In practice, however, the boundaries change, and it is very important to trace these changes. In order to detect these changes, we need to place the sensors near the boundary, so that these sensors will be able to detect how fast the boundary moves.

\section{How to Optimally Place Sensors Near the INTER-ZONE BOUNDARY: TOWARDS THE EXACT FORMULATION OF THE PROBLEM}

Sensors at the boundary. We assume that the boundary is reasonably smooth, so that for some reasonably large (and known) value $\ell$, if we place sensors at distance $\ell$ from each other, we will get a pretty good picture of the whole boundary.

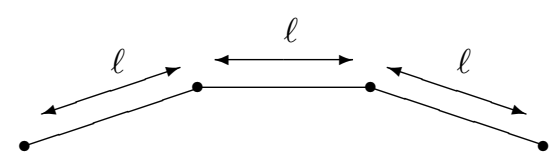

Since each sensor is located at a distance $\ell$ from the previous one, to cover the boundary of total length $L$, we need $L / \ell$ sensors.

We need sensors at different distances from the boundary. In practice, the boundary moves. As a result, after some time, a sensor line that covers the current location of the boundary, will become far away from it and not cover the boundary anymore. To cover the boundary for a long term, we therefore need to place several lines of sensors - to cover not only the current boundary, but also its future locations.

Each sensor line requires $L / \ell$ sensors. So, if we place $k$ sensor lines, we thus place a total of $k \cdot(L / \ell)$ sensors. Let $N$ denote the total number of sensors that our budget can afford. Under this budget restriction, the number of sensor lines $k$ that we can afford can be determined by the condition $k \cdot(L / \ell)=N$. Thus, we can place $k=N /(L / \ell)$ sensor lines.

It is reasonable to assume that we have a rough estimate $V_{0}$ of the velocity with which the boundary moves. We plan the sensor network to be in place for a certain period of time, e.g., $T$ years. During this time, the boundary will move a distance $D=V_{0} \cdot T$. So, to cover all the distances between 0 and $D$, we must place $k$ sensor lines at $k$ different distances from the current border.

Similarly to the above, it is reasonable to select these distances to be equally spaced, i.e., to have sensor lines at distance $0, d=D / k, 2 \cdot d, 3 \cdot d, \ldots$, from the boundary.

How to arrange different sensor lines relative to each other? For each distance $i \cdot d, i=0,1, \ldots$, we place the corresponding sensors on a line which is parallel to the border and which is located at this distance from the border. On this line, the sensors are equally spaced, with a distance $\ell$ between two neighboring sensors. Once we determine a place for one of the sensors on this line, the location of other sensors from this line will be fully determined - namely, we place sensors on this line at distances $\ell, 2 \cdot \ell, \ldots$, from this original sensor.

To make this arrangement more specific, let us start with such an equally spaced arrangement of sensors on the original boundary. In this arrangement, let us pick two neighboring sensors at distance $\ell$ from each other. For each sensor line, we can then select the segment parallel to the segment between these two sensors. This segment is also of length $\ell$, so on this segment, each sensor line has exactly one sensor. Once the locations of all these sensors is fixed, the location of all other sensors is uniquely determined - on each sensor line, we place sensors on this line at distances $\ell, 2 \cdot \ell, \ldots$, from the sensor from this segment. Thus, to fully determine the sensor configuration, we must decide how to place sensors within this segment.

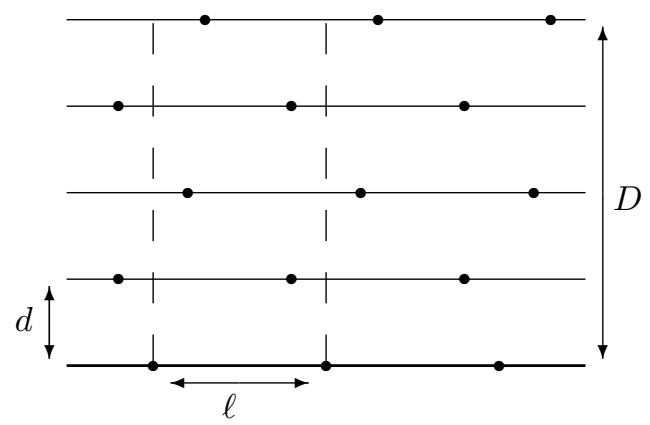

How to place sensors within a segment: selecting an optimal path. When we place sensors, we need to physically travel from one sensor to the next one. We are talking about sensors in a remote area, where travel is difficult. It is therefore reasonable to place sensor in such a way as to minimize the total length of the path connecting all these sensors.

Often, sensors require periodic check-up and maintenance. In this case, in addition to the original sensor placement, we periodically need to re-visit these sensor locations. In this case, it is even more important to minimize the sensor-connecting path.

Let us formulate the problem of selecting the path in precise terms. Each sector is obtained from the previous one by a shift by $\ell$, so it makes sense to repeat the same path in every sector. Among other sensors, we need to visit all the sensors along the original boundary, so let us consider the part of the path that starts with a sensor $S$ at the original boundary and ends us at the next sensor $S^{\prime}$ on this boundary.

We also need to visit sensors which are farthest away - at distance $D$ - from the original boundary. Thus, our sensorvisiting path must start from a point on the original boundary, go to a point $F$ on the line at distance $D$, and then go back: 


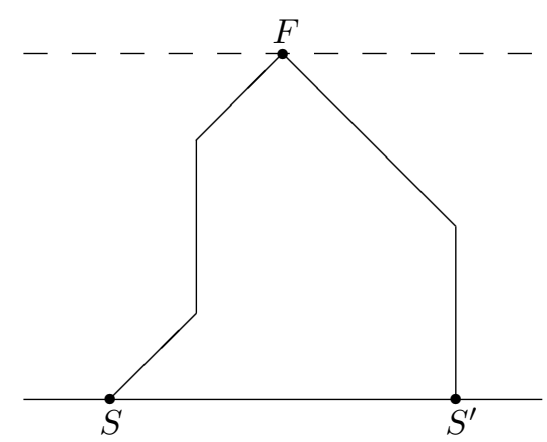

Out of all paths with this property, we must select the shortest one.

\section{AnAlysis of the PROBlem}

First conclusion: the desired shortest path must consist of two straight segments. We need to go from the location $S$ of the first sensor on the original boundary to the location $F$ of a sensor on the farthest sensor line, and then back to the location $S^{\prime}$ of the next sensor on the original boundary. If the points $S$ and $F$ are fixed, then the shortest path from $S$ to $F$ is the straight line. Similarly, the shortest path from $F$ to $S^{\prime}$ is also a straight line. Thus, the shortest path must consist of two straight-line segments: a straight line from $S$ to $F$ and a straight line from $F$ to $S^{\prime}$ :

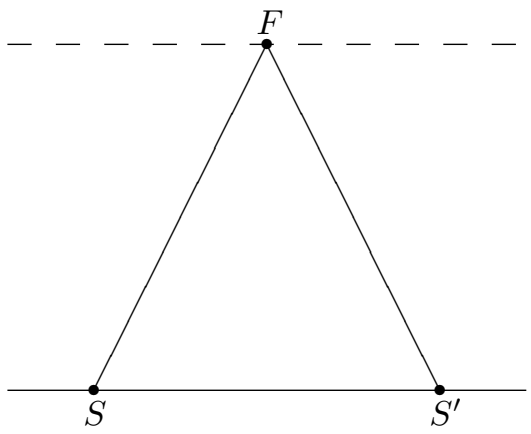

How to select the optimal location of the farthest sensor. In the above two-segment configuration, the sensor locations $S$ and $S^{\prime}$ are fixed. Thus, to finalize the path, we must select an appropriate location of the point $F$. We must select it from the condition that the resulting path $S F S^{\prime}$ is the shortest. Let us solve the corresponding optimization problem. Let $P$ denote the projection of the point $F$ onto the original boundary. Let $x$ denote the distance between the sensor location $S$ and this projection $P$. Then, since the distance between the sensors $S$ and $S^{\prime}$ is equal to $\ell$, the distance $P S^{\prime}$ is equal to $\ell-x$ :

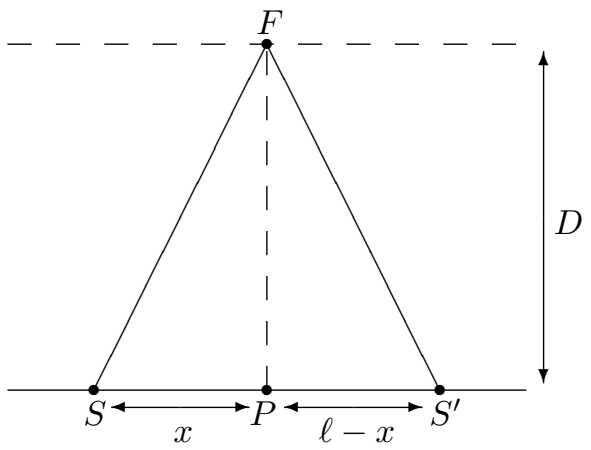

Due to Pythagoras theorem, the length of the segment $S F$ is $\sqrt{D^{2}+x^{2}}$, and the length of the segment $F S^{\prime}$ is equal to $\sqrt{D^{2}+(\ell-x)^{2}}$. Thus, to find the optimal location of the sensor $F$, we must minimize the overall length $p$ of the path $S F S^{\prime}$ :

$$
p=\sqrt{D^{2}+x^{2}}+\sqrt{D^{2}+(\ell-x)^{2}} .
$$

Differentiating this expression and equating the derivative to 0 , we get

$$
\frac{x}{\sqrt{D^{2}+x^{2}}}-\frac{\ell-x}{\sqrt{D^{2}+(\ell-x)^{2}}}=0,
$$

i.e., equivalently,

$$
\frac{x}{\sqrt{D^{2}+x^{2}}}=\frac{\ell-x}{\sqrt{D^{2}+(\ell-x)^{2}}} .
$$

One can easily see that the left-hand side of this formula (3) is the cosine $\cos (\alpha)$ of the angle $\alpha=\angle F S P$, and its right-hand side is the cosine $\cos (\beta)$ of the angle $\beta=\angle F S^{\prime} P$ :

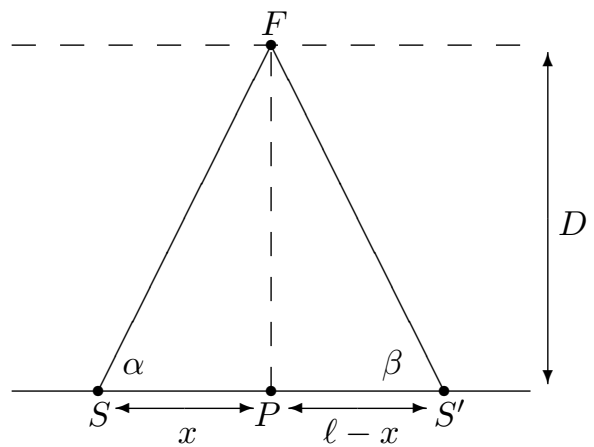

The two angles $\alpha$ and $\beta$ has equal cosines, so they are equal to each other. Thus, the triangle $S F S^{\prime}$ is an isosceles triangle and therefore, its sides $S F$ and $S^{\prime} F$ are also equal to each other. Therefore, the projections $S P$ and $P S^{\prime}$ are also equal, i.e., $x=\ell-x$, and $x=\ell / 2$.

The resulting optimal path. As a result, we get the following optimal path: starting from the location $S$, we take a straight line to a point $F$ which is located on the farthest sensor line midway between $S$ and the next sensor $S^{\prime}$. From that $F$, we take a straight line path back to $S^{\prime}$, etc. As a result, we get the following "see-saw" path:

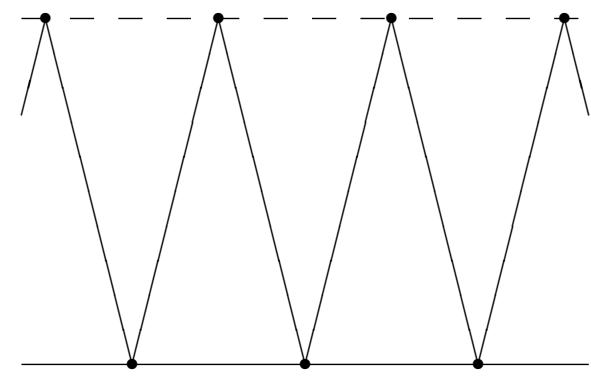

Where to place sensors along the path? Within each segment, the path intersect each sensor line twice, so we can place a sensor either on the ascending or on the descending parts of the path: 


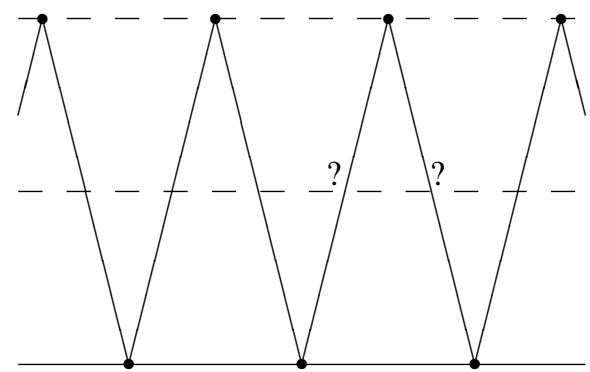

Similarly to the above, it makes sense to place the sensors in such a way that they are distributed along the path as uniformly as possible. Thus, we can, e.g., place sensors on the evennumbered sensor lines on the ascending path, and sensors from the odd-numbered sensor lines on the descending path:

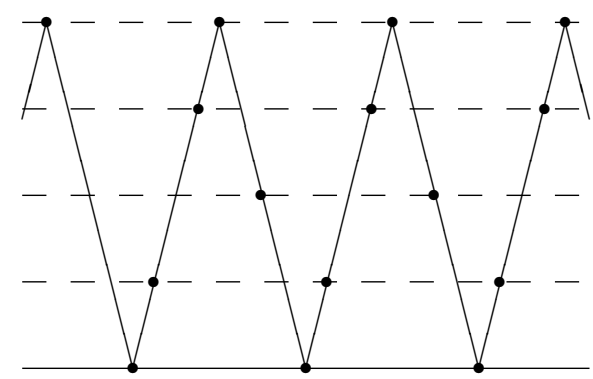

\section{Conclusion: Resulting Optimal Placement}

We assume:

- to maintain desired accuracy, we need to place sensor at distance at most $\ell$ along the boundary;

- that during the sensor lifetime, the boundary will move by the distance $D$; and

- that, based on the available (or affordable) number of sensors, we can place the sensors along $k$ sensor lines, located at distance $0, d=D / k, 2 d, \ldots$, from the original boundary.

Our objectives are to minimize the path that we need to traverse to place and to maintain the sensor, and to maintain the most accurate (hence, homogeneous) spatial coverage at any given moment of time. To satisfy these objectives, we place the sensor at equal distance $\ell$ along the original sensor boundary, and then place other sensors along the see-saw path that goes from every sensor on the original boundary to the farthest (distance $D$ ) sensor line and then back - at the exact same angle.

Sensors from the even-numbered sensors are then placed on the ascending part of the sensor-connecting path, and sensors from the odd-numbered sensors are then placed on the descending part of the sensor-connecting path.

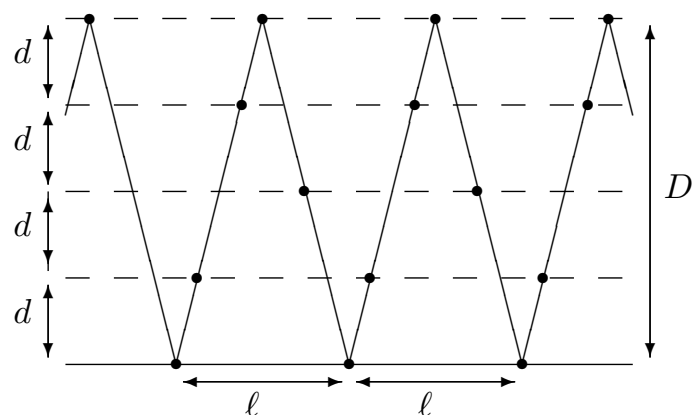

ACKNOWLEDGMENT

This work was supported in part by the National Science Foundation grants HRD-0734825 and DUE-0926721, by Grant 1 T36 GM078000-01 from the National Institutes of Health, by Grant MSM 6198898701 from MŠMT of Czech Republic, and by Grant 5015 "Application of fuzzy logic with operators in the knowledge based systems" from the Science and Technology Centre in Ukraine (STCU), funded by European Union.

The authors are thankful to the anonymous referees for valuable suggestions.

\section{REFERENCES}

[1] H. T. Nguyen, O. Kosheleva, V. Kreinovich, and S. Ferson, "TradeOff Between Sample Size and Accuracy: Case of Dynamic Measurements under Interval Uncertainty", In: V.-N. Huynh, Y. Nakamori, H. Ono, J. Lawry, V. Kreinovich, and H. T. Nguyen (eds.), Interval/Probabilistic Uncertainty and Non-Classical Logics, Springer-Verlag, Berlin-Heidelberg-New York, 2008, pp. 45-56.

[2] H. T. Nguyen, O. Kosheleva, V. Kreinovich, and S. Ferson, "TradeOff Between Sample Size and Accuracy: Case of Measurements under Interval Uncertainty", International Journal of Approximate Reasoning, 2009, Vol. 50, No. 8, pp. 1164-1176.

[3] H. T. Nguyen and V. Kreinovich, "Trade-Off Between Sample Size and Accuracy: Case of Static Measurements under Interval Uncertainty", In: V.-N. Huynh, Y. Nakamori, H. Ono, J. Lawry, V. Kreinovich, and H. T. Nguyen (eds.), Interval/Probabilistic Uncertainty and Non-Classical Logics, Springer-Verlag, Berlin-Heidelberg-New York, 2008, pp. 32-44. 